\title{
Stability Boundary Characterization of Nonlinear Autonomous Dynamical Systems in the Presence of Saddle-Node Equilibrium Points ${ }^{1}$
}

F.M. AMARAL 2 , Departamento de Ensino, Instituto Federal de Educação, Ciência e Tecnologia Bahia, Campus Eunápolis, 45.822-000 Eunápolis, BA, Brasil.

L.F.C. ALBERTO 3 , Departamento de Engenharia Elétrica, Escola de Engenharia Elétrica de São Carlos, Universidade de São Paulo, 13.566-590 São Carlos, SP, Brasil.

\begin{abstract}
A dynamical characterization of the stability boundary for a fairly large class of nonlinear autonomous dynamical systems is developed in this paper. This characterization generalizes the existing results by allowing the existence of saddlenode equilibrium points on the stability boundary. The stability boundary of an asymptotically stable equilibrium point is shown to consist of the stable manifolds of the hyperbolic equilibrium points on the stability boundary and the stable, stable center and center manifolds of the saddle-node equilibrium points on the stability boundary.
\end{abstract}

Keywords. Stability Region, Stability Boundary, Saddle-Node Equilibrium Point.

\section{Introduction}

Asymptotically stable equilibrium points of many practical nonlinear dynamical systems are not globally stable. As a consequence, the determination of stability regions (region of attraction or basin of attraction) of asymptotically stable equilibrium points is a fundamental problem in nonlinear system theory [10] with great importance in several applications $[19,17,4]$. The exact stability region is of difficult determination and, over the last thirty years, a great number of methods were proposed for estimating the stability region of attractors of nonlinear dynamical systems [16].

Some recent methods, such as those developed in [8] and [4], explore a topological characterization of the stability boundary (the boundary of the stability region) to obtain good estimates of the stability region. Therefore, developing characterizations of stability boundaries of nonlinear dynamical systems is of fundamental importance for developing efficient tools for stability region estimation.

\footnotetext{
${ }^{1}$ The authors would like to thank FAPESP (research project 2010/00574-9) and CNPq (Conselho Nacional de Desenvolvimento Científico e Tecnológico) for the financial support.

${ }^{2}$ fabiolo@ifba.edu.br.

${ }^{3}$ lfcalberto@usp.br.
} 
Under some reasonable assumptions, the stability boundary of an asymptotically stable equilibrium point was characterized in terms of the stable manifolds of a set of unstable equilibria (and/or closed orbits) lying on this boundary [6]. These existing characterizations of stability boundaries were proved under the key assumption that all the equilibrium points on the stability boundary are hyperbolic. A first generalization of this characterization appeared in [2] by considering the existence of a particular type of non-hyperbolic equilibrium point, the so called type-zero saddle-node equilibrium point, on the stability boundary. In this paper, a further generalization of this characterization of the stability boundary is developed by allowing the presence of any type of saddle-node equilibrium point on the stability boundary. The characterization of the stability boundary in the presence of saddlenode equilibrium points is of fundamental importance for studying stability region bifurcations [3].

In this paper, a complete characterization of the stability boundary of nonlinear dynamical systems possessing saddle-node equilibrium points on it is presented. It is shown that the stability boundary consists of the stable manifolds of the hyperbolic equilibrium points on the stability boundary and the stable, stable center and center manifolds of the saddle-node equilibrium points on the stability boundary. Necessary and sufficient conditions for a saddle-node equilibrium point lying on the stability boundary are also developed.

\section{Preliminaries}

In this section, some classical concepts of the theory of dynamical systems are reviewed. In particular, an overview of the main features of the dynamic behavior of a system in the neighborhood of a specific type of non-hyperbolic equilibrium point, the saddle-node equilibrium point, is presented. More details on the content explored in this section can be found in [11, 21, 18].

Consider the nonlinear autonomous dynamical system

$$
\dot{x}=f(x)
$$

where $x \in \mathbb{R}^{n}$. One assumes that $f: \mathbb{R}^{n} \rightarrow \mathbb{R}^{n}$ is a vector field of class $C^{r}$ with $r \geq 2$. The solution of (2.1) starting at $x$ at time $t=0$ is denoted by $\varphi(t, x)$. The map $t \rightarrow \varphi(t, x)$ defines in $\mathbb{R}^{n}$ a curve passing through $x$ at $t=0$ that is called trajectory or orbit of (2.1) through $x$. If $M$ is a set of initial conditions, then $\varphi(t, M)$ denotes the set $\{\varphi(t, x), x \in M\}=\bigcup_{x \in M} \varphi(t, x)$. A set $S \in \mathbb{R}^{n}$ is said to be an invariant set of (2.1) if every trajectory of (2.1) starting in $S$ remains in $S$ for all $t$.

\subsection{Hyperbolic equilibrium points}

A point $x^{*} \in \mathbb{R}^{n}$ is an equilibrium point of (2.1) if $f\left(x^{*}\right)=0$. An equilibrium point $x^{*}$ is said to be hyperbolic if none of the eigenvalues of the Jacobian matrix $D f\left(x^{*}\right)$ of $f(x)$, calculated at the equilibrium point $x^{*}$, has real part equal to zero. Moreover, a hyperbolic equilibrium point $x^{*}$ is of type- $k$ if the Jacobian matrix $D f\left(x^{*}\right)$ possesses $k$ eigenvalues with positive real part and $n-k$ eigenvalues with negative real part. 
Let $x^{*}$ be a hyperbolic equilibrium point of the nonlinear dynamical system (2.1). Then there exists a neighborhood $U$ of $x^{*}$ and local stable and unstable manifolds [13], $W_{l o c}^{s}\left(x^{*}\right)=\left\{x \in U: \varphi(t, x) \rightarrow x^{*}\right.$ as $\left.t \rightarrow \infty\right\}$ and $W_{l o c}^{u}\left(x^{*}\right)=\{x \in$ $U: \varphi(t, x) \rightarrow x^{*}$ as $\left.t \rightarrow-\infty\right\}$ with the following properties: (i) they have the same dimensions as those of the eigenspaces $E^{s}$ and $E^{u}$ of the linearized system $\dot{z}=D f\left(x^{*}\right) z$, therefore the sum of the dimension of $W_{l o c}^{s}\left(x^{*}\right)$ and of $W_{l o c}^{u}\left(x^{*}\right)$ equals the dimension of the state space; (ii) they are tangent to $E^{s}$ and $E^{u}$ at $x^{*}$; and (iii) they are as smooth as function $f$.

The stable manifold $W^{s}\left(x^{*}\right)$ and the unstable manifold $W^{u}\left(x^{*}\right)$, which are invariant sets, are obtained by letting the points in $W_{l o c}^{s}\left(x^{*}\right)$ to flow backwards in time and the points in $W_{l o c}^{u}\left(x^{*}\right)$ to flow forwards in time [22]:

$$
W^{s}\left(x^{*}\right)=\bigcup_{t \leq 0} \varphi\left(t, W_{l o c}^{s}\left(x^{*}\right)\right) \quad W^{u}\left(x^{*}\right)=\bigcup_{t \geq 0} \varphi\left(t, W_{l o c}^{u}\left(x^{*}\right)\right) .
$$

\subsection{Saddle-Node equilibrium points}

In this section, a specific type of non-hyperbolic equilibrium point, namely saddlenode equilibrium point, is studied. In particular, the dynamical behavior in a neighborhood of the equilibrium is investigated, including the asymptotic behavior of solutions in the invariant local manifolds.

Definition 2.1. [21](Saddle-Node Equilibrium Point): A non-hyperbolic equilibrium point $p \in \mathbb{R}^{n}$ of (2.1) is called a saddle-node equilibrium point if the following conditions are satisfied:

(i) $D_{x} f(p)$ has a unique simple null eigenvalue and none of the other eigenvalues have real part equal to zero.

(ii) $w\left(D_{x}^{2} f(p)(v, v)\right) \neq 0$,

with $v$ as the right eigenvector and $w$ the left eigenvector associated with the null eigenvalue.

Saddle-node equilibrium points can be classified in types according to the number of eigenvalues of $D_{x} f(p)$ with positive real part.

Definition 2.2. (Saddle-Node Equilibrium Type): A saddle-node equilibrium point $p$ of (2.1), is called a type- $k$ saddle-node equilibrium point if $D_{x} f(p)$ has $k$ eigenvalues with positive real part and $n-k-1$ with negative real part.

If $p$ is a saddle-node equilibrium point of (2.1), then there exist invariant local manifolds $W_{l o c}^{s}(p), W_{l o c}^{c s}(p), W_{l o c}^{c}(p), W_{l o c}^{u}(p)$ and $W_{l o c}^{c u}(p)$ of class $C^{r}$, tangent to $E^{s}$, $E^{c} \oplus E^{s}, E^{c}, E^{u}$ and $E^{c} \oplus E^{u}$ at $p$, respectively [13]. These manifolds are respectively called stable, stable center, center, unstable and unstable center manifolds. The stable and unstable manifolds are unique, but the stable center, center and unstable center manifolds may not be.

If $p$ is a saddle-node equilibrium point, then the following properties hold [21]:

(1) $p$ is a type-0 saddle-node equilibrium point of (2.1): 
(i) The $(n-1)$-dimensional local stable manifold $W_{l o c}^{s}(p)$ of $p$ exists, is unique, and if $q \in W_{l o c}^{s}(p)$ then $\varphi(t, q) \longrightarrow p$ as $t \longrightarrow+\infty$.

(ii) The unidimensional local center manifold $W_{l o c}^{c}(p)$ of $p$ can be splitted in two invariant submanifolds:

$$
W_{l o c}^{c}(p)=W_{l o c}^{c^{-}}(p) \cup W_{l o c}^{c^{+}}(p)
$$

where $q \in W_{l o c}^{c^{-}}(p)$ implies $\varphi(t, q) \longrightarrow p$ as $t \longrightarrow+\infty$ and $q \in W_{l o c}^{c^{+}}(p)$ implies $\varphi(t, q) \longrightarrow p$ as $t \longrightarrow-\infty$. Moreover, $W_{l o c}^{c^{+}}(p)$ is unique while $W_{l o c}^{c^{-}}(p)$ is not.

(2) $p$ is a type- $k$ saddle-node equilibrium point of (2.1) with $1 \leq k \leq n-2$ :

(i) The $k$-dimensional local unstable manifold $W_{l o c}^{u}(p)$ of $p$ exists, is unique, and if $q \in W_{l o c}^{u}(p)$ then $\varphi(t, q) \longrightarrow p$ as $t \longrightarrow-\infty$.

(ii) The $(n-k-1)$-dimensional local stable manifold $W_{\text {loc }}^{s}(p)$ of $p$ exists, is unique, and if $q \in W_{l o c}^{s}(p)$ then $\varphi(t, q) \longrightarrow p$ as $t \longrightarrow+\infty$.

(iii) The $(n-k)$-dimensional local stable center manifold $W_{l o c}^{c s}(p)$ of $p$ can be splitted in two invariant submanifolds:

$$
W_{l o c}^{c s}(p)=W_{l o c}^{c s^{-}}(p) \cup W_{l o c}^{c s^{+}}(p)
$$

where $q \in W_{l o c}^{c s^{-}}(p)$ implies $\varphi(t, q) \longrightarrow p$ as $t \longrightarrow+\infty$. The local stable center manifold $W_{l o c}^{s}(p)$ is contained in $W_{l o c}^{c s^{-}}(p)$, moreover, $W_{l o c}^{c s^{-}}(p)$ is unique while $W_{l o c}^{c s^{+}}(p)$ is not.

(iv) The $(k+1)$-dimensional local unstable center manifold $W_{l o c}^{c u}(p)$ of $p$ can be splitted in two invariant submanifolds:

$$
W_{l o c}^{c u}(p)=W_{l o c}^{c u^{-}}(p) \cup W_{l o c}^{c u^{+}}(p)
$$

where $q \in W_{l o c}^{c u^{+}}(p)$ implies $\varphi(t, q) \longrightarrow p$ as $t \longrightarrow-\infty$. The local unstable center manifold $W_{l o c}^{u}(p)$ is contained in $W_{l o c}^{c u^{+}}(p)$, moreover, $W_{l o c}^{c u^{+}}(p)$ is unique while $W_{l o c}^{c u^{-}}(p)$ is not.

(3) $p$ is a type- $(n-1)$ saddle-node equilibrium point of $(2.1)$ :

(i) The $(n-1)$-dimensional local unstable manifold $W_{l o c}^{u}(p)$ of $p$ exists, is unique, and if $q \in W_{l o c}^{u}(p)$ then $\varphi(t, q) \longrightarrow p$ as $t \longrightarrow-\infty$.

(ii) The unidimensional local center manifold $W_{l o c}^{c}(p)$ of $p$ can be splitted in two invariant submanifolds:

$$
W_{l o c}^{c}(p)=W_{l o c}^{c^{-}}(p) \cup W_{l o c}^{c^{+}}(p)
$$

where $q \in W_{l o c}^{c^{-}}(p)$ implies $\varphi(t, q) \longrightarrow p$ as $t \longrightarrow+\infty$ and $q \in W_{l o c}^{c^{+}}(p)$ implies $\varphi(t, q) \longrightarrow p$ as $t \longrightarrow-\infty$. Moreover, $W_{l o c}^{c^{-}}(p)$ is unique while $W_{l o c}^{c^{+}}(p)$ is not. 


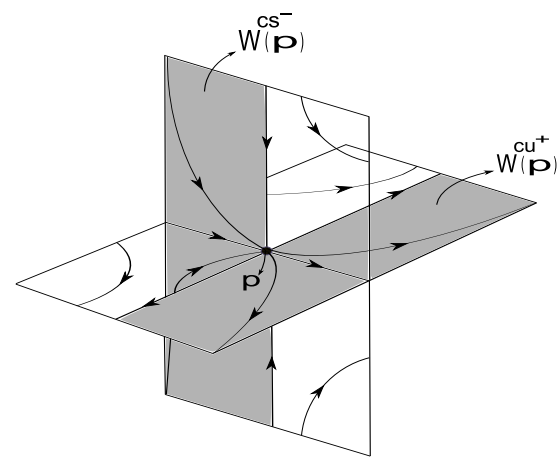

Figure 1: Manifolds $W_{l o c}^{c s^{-}}(p)$ and $W_{l o c}^{c u}(p)$ for a type-1 saddle-node equilibrium point $p$ of system (2.1) in $\mathbb{R}^{3}$.

Although the stable and unstable manifolds of a hyperbolic equilibrium point are defined by extending the local manifolds through the flow, this technique cannot be applied to general non-hyperbolic equilibrium points. However, in the particular case of a saddle-node equilibrium point $p$, one still can define the global manifolds $W^{s}(p), W^{u}(p), W^{c^{+}}(p), W^{c^{-}}(p), W^{c s^{-}}(p)$ and $W^{c u^{+}}(p)$ extending the local manifolds $W_{l o c}^{s}(p), W_{l o c}^{u}(p), W_{l o c}^{c^{+}}(p), W_{l o c}^{c^{-}}(p), W_{l o c}^{c s^{-}}(p)$ and $W_{l o c}^{c u^{+}}(p)$ through the flow as follows:

$$
\begin{aligned}
& W^{s}(p):=\bigcup_{t \leq 0} \varphi\left(t, W_{l o c}^{s}(p)\right) \quad W^{u}(p):=\bigcup_{t \geq 0} \varphi\left(t, W_{l o c}^{u}(p)\right) \\
& W^{c s^{-}}(p):=\bigcup_{t \leq 0} \varphi\left(t, W_{l o c}^{c s^{-}}(p)\right) \quad W^{c u^{+}}(p):=\bigcup_{t \geq 0} \varphi\left(t, W_{l o c}^{c u^{+}}(p)\right) \\
& W^{c^{-}}(p):=\bigcup_{t \leq 0} \varphi\left(t, W_{l o c}^{c^{-}}(p) \text { and } W^{c^{+}}(p):=\bigcup_{t \geq 0} \varphi\left(t, W_{l o c}^{c^{+}}(p)\right) .\right.
\end{aligned}
$$

This extension is justified by the aforementioned invariance and the asymptotic behavior of the local manifolds $W_{l o c}^{s}(p), W_{l o c}^{u}(p), W_{l o c}^{c^{+}}(p), W_{l o c}^{c^{-}}(p), W_{l o c}^{c s^{-}}(p)$ and $W_{l o c}^{c u^{+}}(p)$, see items (1), (2) and (3) above. Figure 1 illustrates the manifolds $W_{l o c}^{c s^{-}}(p)$ and $W_{l o c}^{c u^{+}}(p)$ for a type-1 saddle-node equilibrium point $p$.

\subsection{Stability Region}

Suppose $x^{s}$ is an asymptotically stable equilibrium point of (2.1). The stability region (or region of attraction) of $x^{s}$ is the set

$$
A\left(x^{s}\right)=\left\{x \in \mathbb{R}^{n}: \varphi(t, x) \rightarrow x^{s} \text { as } t \rightarrow \infty\right\},
$$

of all initial conditions $x \in \mathbb{R}^{n}$ whose trajectories converge to $x^{s}$ when $t$ tends to infinity. 
The stability region $A\left(x^{s}\right)$ is an open and invariant set. Its closure $\overline{A\left(x^{s}\right)}$ is invariant and the stability boundary $\partial A\left(x^{s}\right)$, the topological boundary of $A\left(x^{s}\right)$, is a closed and invariant set.

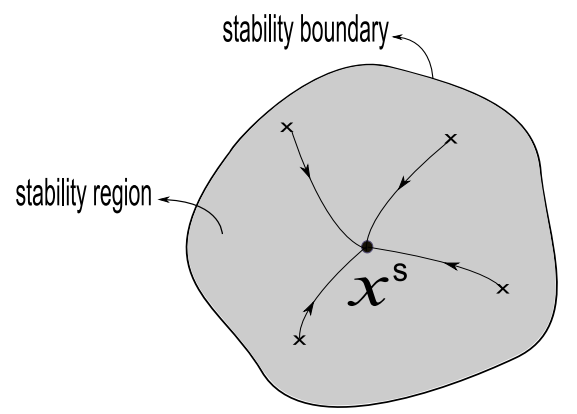

Figure 2: Stability region and stability boundary of an asymptotically stable equilibrium point $x^{s}$.

\section{Hyperbolic Equilibrium Points on the Stability Boundary}

In this section, an overview of the existing body of theory about the stability boundary characterization of nonlinear dynamical systems is presented. The unstable equilibrium points that lie on the stability boundary $\partial A\left(x^{s}\right)$ play an essential role in the stability boundary characterization.

Let $x^{s}$ be a hyperbolic asymptotically stable equilibrium point of (2.1) and consider the following assumptions:

(A1) All the equilibrium points on $\partial A\left(x^{s}\right)$ are hyperbolic.

(A2) Every trajectory on $\partial A\left(x^{s}\right)$ approaches an equilibrium point as $t \rightarrow+\infty$.

Assumption (A1) is a generic property of dynamical systems in the form of (2.1). In other words, it is satisfied for almost all dynamical systems in the form of (2.1) and, in practice, does not need to be verified. On the contrary, assumption (A2) is not a generic property of dynamical systems and needs to be checked. In spite of that, many nonlinear dynamical systems satisfy this property. In particular, the existence of an energy function is a sufficient condition to guarantee the satisfaction of (A2) [6].

Under assumptions(A1)and(A2), the next theorem provides a complete characterization of the stability boundary $\partial A\left(x^{s}\right)$. It asserts that the stability boundary $\partial A\left(x^{s}\right)$ is the union of the stable manifolds of the unstable equilibrium points on $\partial A\left(x^{s}\right)$.

Theorem 3.1. (Stability boundary characterization)[6] Let $x^{s}$ be an asymptotically stable equilibrium point of (2.1) and $A\left(x^{s}\right)$ be its stability region. If as- 
sumptions $(A 1)$ and $(A 2)$ are satisfied, then:

$$
\partial A\left(x^{s}\right) \subseteq \bigcup_{i} W^{s}\left(x^{i}\right)
$$

where $x^{i}, i=1,2, \ldots$ are the hyperbolic equilibrium points on the stability boundary $\partial A\left(x^{s}\right)$. If, in addition, $W^{u}\left(x^{i}\right) \cap A\left(x^{s}\right) \neq \emptyset, i=1,2, \ldots$, then

$$
\partial A\left(x^{s}\right)=\bigcup_{i} W^{s}\left(x^{i}\right)
$$

Theorem 3.1 provides a complete stability boundary characterization of system (2.1) under assumptions $(A 1)$ and $(A 2)$. Sufficient conditions to guarantee that $W^{u}\left(x^{i}\right) \cap A\left(x^{s}\right) \neq \emptyset$ when a hyperbolic equilibrium point $x^{i} \in \partial A\left(x^{s}\right)$ are also provided in $[6]$.

\section{Saddle-Node Equilibrium Points on the Stability Boundary}

In the presence of non hyperbolic equilibrium points on the stability boundary, Theorem 3.1 is not valid. In this section, a generalization of the results of Theorem 3.1 about stability boundary characterization is developed. We study the stability boundary characterization when assumption $(A 1)$ is violated. In particular, a complete characterization of the stability boundary is developed when a particular type of non-hyperbolic equilibrium point, the so called saddle-node equilibrium point, lies on the the stability boundary $\partial A\left(x^{s}\right)$.

Next theorem offers necessary and sufficient conditions to guarantee that a saddle-node equilibrium point lies on the stability boundary in terms of the properties of its stable, unstable and center manifolds.

Theorem 4.1. (Saddle-Node Equilibrium Point on the Stability Boundary): Let $p$ be a saddle-node equilibrium point of (2.1). Suppose also, the existence of an asymptotically stable equilibrium point $x^{s}$ and let $A\left(x^{s}\right)$ be its stability region. Then the following holds:

(i) If $p$ is a type-0 saddle-node equilibrium point, then:

$$
\begin{aligned}
& p \in \partial A\left(x^{s}\right) \Leftrightarrow\left(W^{c^{+}}(p)-\{p\}\right) \cap \overline{A\left(x^{s}\right)} \neq \emptyset \\
& p \in \partial A\left(x^{s}\right) \Leftrightarrow\left(W^{s}(p)-\{p\}\right) \cap \partial A\left(x^{s}\right) \neq \emptyset .
\end{aligned}
$$

(ii) If $p$ is a type- $k$ saddle-node equilibrium point, $1 \leq k \leq n-2$, then:

$$
\begin{aligned}
& p \in \partial A\left(x^{s}\right) \Leftrightarrow\left(W^{c u^{+}}(p)-\{p\}\right) \cap \overline{A\left(x^{s}\right)} \neq \emptyset \\
& p \in \partial A\left(x^{s}\right) \Leftrightarrow\left(W^{s}(p)-\{p\}\right) \cap \partial A\left(x^{s}\right) \neq \emptyset .
\end{aligned}
$$


(iii) If $p$ is a type- $(n-1)$ saddle-node equilibrium point, then:

$$
p \in \partial A\left(x^{s}\right) \Leftrightarrow\left(W^{c u^{+}}(p)-\{p\}\right) \cap \overline{A\left(x^{s}\right)} \neq \emptyset .
$$

Proof. (i) The proof of item $(i)$ can be found in [2].

(ii) $(\Longleftarrow)$ Suppose first that $\left(W^{c u+}(p)-\{p\}\right) \cap \overline{A\left(x^{s}\right)} \neq \emptyset$. Then there exists $x \in W^{c u^{+}}(p) \cap \overline{A\left(x^{s}\right)}$. Note that $\varphi(t, x) \longrightarrow p$ as $t \longrightarrow-\infty$. On the other hand, set $\overline{A\left(x^{s}\right)}$ is invariant, thus $\varphi(t, x) \in \overline{A\left(x^{s}\right)}$ for all $t \leq 0$. As a consequence, $p \in \overline{A\left(x^{s}\right)}$. Since $p \notin A\left(x^{s}\right)$, we have that $p \in\left(\mathbb{R}^{n}-A\left(x^{s}\right)\right)$. Therefore, $p \in$ $\partial A\left(x^{s}\right)$. Now if $\left(W^{s}(p)-\{p\}\right) \cap \partial A\left(x^{s}\right) \neq \emptyset$ then there exists at least a point $x \in\left(W^{s}(p)-\{p\}\right) \cap \partial A\left(x^{s}\right)$. Moreover, $\varphi(t, x) \rightarrow p$ as $t \rightarrow \infty$. Since $\partial A\left(x^{s}\right)$ is closed and invariant, then $p \in \partial A\left(x^{s}\right)$.

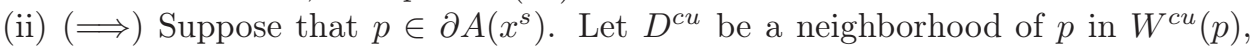
whose boundary $\partial D^{c u}$ is transversal to the vector field $f$ on $W^{c u+}$, and define $D^{c u^{+}}:=D^{c u} \cap W^{c u^{+}}(p)$. Consider $L_{\epsilon}^{c u^{+}}=\left\{x \in \mathbb{R}^{n}: d\left(x, \partial D^{c u^{+}}\right)<\epsilon\right\}$ for some $\epsilon>0$. As a consequence of $\lambda$-lemma for non-hyperbolic equilibrium points [18], we can take a neighborhood $U$ of $p$ such that $\cup_{t \leq 0} \varphi\left(t, L_{\epsilon}^{c u^{+}}\right) \supset\left(U-\left\{W^{c s^{-}}(p)\right\}\right)$. Since $p \in \partial A\left(x^{s}\right)$, we have that $U \cap A\left(x^{s}\right) \neq \emptyset$. On the other hand, as $W^{c s^{-}}(p) \cap A\left(x^{s}\right)=\emptyset$, we can affirm that $\left(U-\left\{W^{c s^{-}}(p)\right\}\right) \cap A\left(x^{s}\right) \neq \emptyset$. Thus, there exists a point $q^{*} \in L_{\epsilon}^{c u^{+}}$ and a time $t^{*}$ such that $\varphi\left(t^{*}, q^{*}\right) \in A\left(x^{s}\right)$. Since $A\left(x^{s}\right)$ is invariant, we have that $q^{*} \in A\left(x^{s}\right)$. Since $\epsilon$ can be chosen arbitrarily small, we can find a sequence of points $\left\{q_{i}^{*}\right\}$ with $q_{i}^{*} \in A\left(x^{s}\right)$ for all $i=1,2, \ldots$ such that $d\left(q_{i}^{*}, \partial D^{c u^{+}}\right) \rightarrow 0$ as $i \longrightarrow+\infty$. By construction, this sequence is bounded, so has a convergent subsequence, that is, $q_{i_{j}}^{*} \rightarrow q$ as $i_{j} \rightarrow+\infty$. Thus, $d\left(q_{i_{j}}^{*}, \partial D^{c u^{+}}\right) \rightarrow d\left(q, \partial D^{c u^{+}}\right)$as $i_{j} \longrightarrow+\infty$ and consequently $q \in \overline{\partial D^{c u^{+}}} \subset\left(W^{c u^{+}}(p)-\{p\}\right)$. Therefore $\left(W^{c u^{+}}(p)-\{p\}\right) \cap \overline{A\left(x^{s}\right)} \neq \emptyset$. The proof that $p \in \partial A\left(x^{s}\right) \Leftrightarrow\left(W^{s}(p)-\{p\}\right) \cap \partial A\left(x^{s}\right) \neq \emptyset$ and of item (iii) is similar to the above proof and will be omitted.

Let $x^{s}$ be an asymptotically stable equilibrium point of (2.1) and consider the following assumption:

(A1 $\left.{ }^{\prime}\right)$ All the equilibrium points on $\partial A\left(x^{s}\right)$ are either hyperbolic or saddle-node equilibrium points.

Under assumptions $\left(A 1^{\prime}\right)$ and $(A 2)$, next theorem offers a complete characterization of the stability boundary of nonlinear autonomous dynamical systems in the presence of saddle-node equilibrium points on the stability boundary $\partial A\left(x^{s}\right)$.

Theorem 4.2. (Stability Boundary Characterization): Let $x^{s}$ be an asymptotically stable equilibrium point of (2.1) and $A\left(x^{s}\right)$ be its stability region. If assumptions $\left(A 1^{\prime}\right)$ and $(A 2)$ are satisfied, then

$$
\partial A\left(x^{s}\right) \subseteq \bigcup_{i} W^{s}\left(x_{i}\right) \bigcup_{j} W^{s}\left(p_{j}\right) \bigcup_{l} W^{c s^{-}}\left(z_{l}\right) \bigcup_{m} W^{c^{-}}\left(q_{m}\right)
$$

where $x^{i}$ are the hyperbolic equilibrium points, $p_{j}$ the type-0 saddle-node equilibrium points, $z_{l}$ the type- $k$ saddle-node equilibrium points, with $1 \leq k \leq n-2$ and $q_{m}$ 
the type- $(n-1)$ saddle-node equilibrium points on $\partial A\left(x^{s}\right), i, j, l, m=1,2, \ldots$ If, in addition, $W^{c^{+}}\left(p_{j}\right) \cap A\left(x^{s}\right) \neq \emptyset$, for all $j=1,2, \ldots$ and the unstable manifolds of all other equilibrium points on the stability boundary $\partial A\left(x^{s}\right)$ intersect the stability region $A\left(x^{s}\right)$, then:

$$
\partial A\left(x^{s}\right)=\bigcup_{i} W^{s}\left(x_{i}\right) \bigcup_{j} W^{s}\left(p_{j}\right) \bigcup_{l} W^{c s^{-}}\left(z_{l}\right) \bigcup_{m} W^{c^{-}}\left(q_{m}\right) .
$$

Proof. If $q \in \partial A\left(x^{s}\right)$, from assumptiom $(A 2)$, we can affirm that $\varphi(t, q) \longrightarrow q^{*}$ for some equilibrium point $q^{*} \in \partial A\left(x^{s}\right)$. From assumptiom $\left(A 1^{\prime}\right)$, we can affirm that $q^{*}$ is either a hyperbolic equilibrium point or a saddle-node equilibrium point, that is, $q^{*}=x_{i}$ or $q^{*}=p_{j}$ or $q^{*}=z_{l}$ or $q^{*}=q_{m}$ for some $i, j, l, m$. Since, the intersection $W^{c^{-}}\left(p_{j}\right) \cap \partial A\left(x^{s}\right)$ is empty [2], we can affirm that $q \in$ $\bigcup_{i} W^{s}\left(x_{i}\right) \bigcup_{j} W^{s}\left(p_{j}\right) \bigcup_{l} W^{c s^{-}}\left(z_{l}\right) \bigcup_{m} W^{c^{-}}\left(q_{m}\right)$. Therefore, $\partial A\left(x^{s}\right) \subseteq \bigcup_{i} W^{s}\left(x_{i}\right)$ $\bigcup_{j} W^{s}\left(p_{j}\right) \bigcup_{l} W^{c s^{-}}\left(z_{l}\right) \bigcup_{m} W^{c^{-}}\left(q_{m}\right)$. In order to prove the other inclusion, we explore the facts that $W^{u}\left(x_{i}\right) \cap A\left(x^{s}\right) \neq \emptyset$ for all $i=1,2, \ldots, W^{u}\left(z_{l}\right) \cap A\left(x^{s}\right) \neq \emptyset$ for all $l=1,2, \ldots, W^{u}\left(q_{m}\right) \cap A\left(x^{s}\right) \neq \emptyset$ for all $m=1,2, \ldots$ and $W^{c+}\left(p_{j}\right) \cap A\left(x_{s}\right) \neq \emptyset$ for all $j=1,2, \ldots$. If $z_{l}$ is a type $k$ saddle-node equilibrium point, with $1 \leq k \leq n-2$, on $\partial A\left(x^{s}\right)$, then, by assumption, $W^{u}\left(z_{l}\right) \cap A\left(x^{s}\right) \neq \emptyset$, then there is $w \in W^{u}\left(z_{l}\right) \cap A\left(x^{s}\right)$. Let $B(w, \epsilon)$ be an open ball with an arbitrarily small radius $\epsilon$ centered at $w$. Radius $\epsilon$ can be chosen arbitrarily small such that $B(w, \epsilon) \subset A\left(x^{s}\right)$. Let $q_{1}$ be an arbitrary point of $W^{c s^{-}}\left(z_{l}\right)$ and consider a disk $D$ of dimension $k$ that is transversal to $W^{c s^{-}}\left(z_{l}\right)$ at $q_{1}$. As a consequence of $\lambda$-lemma for non-hyperbolic equilibrium points [18], there exists an element $z \in D$ and a time $t^{*}>0$ such that $\varphi\left(t^{*}, z\right) \in B(w, \epsilon)$. Since $A\left(x^{s}\right)$ is invariant, we have that $z \in A\left(x^{s}\right)$. Since $\epsilon$ and the disk $D$ can be chosen arbitrarily small, then there exist points of $A\left(x^{s}\right)$ arbitrarily close to $q_{1}$. Therefore $q_{1} \in \overline{A\left(x^{s}\right)}$. Since $W^{c s^{-}}\left(z_{l}\right)$ cannot contain points on $A\left(x^{s}\right)$, $q_{1} \in \partial A\left(x^{s}\right)$. Exploring the fact that $q_{1}$ was arbitrarily taken in $W^{c s^{-}}\left(z_{l}\right)$, we can affirm that $W^{c s^{-}}\left(z_{l}\right) \subset \partial A\left(x^{s}\right)$. Similarly, it can be shown that $W^{s}\left(x_{i}\right) \subset \partial A\left(x^{s}\right)$, $W^{s}\left(p_{j}\right) \subset \partial A\left(x^{s}\right)$ and $W^{c^{-}}\left(q_{m}\right) \subset \partial A\left(x^{s}\right)$, consequently the theorem is proven.

Theorem 4.2 is more general than Theorem 3.1, since assumption $(A 1)$, used in the proof of Theorem 3.1, is relaxed. It also generalizes the results in [2] where only type-zero saddle-node equilibrium points were considered.

\section{Example}

The system of differential equations (5.1) was derived from problems of stability in power systems analysis [9]:

$$
\begin{aligned}
& \dot{x}=1-2,84 \operatorname{sen}(x)-2 \operatorname{sen}(x-y) \\
& \dot{y}=1-3 \operatorname{sen}(y)-2 \operatorname{sen}(y-x)
\end{aligned}
$$

The stability region and stability boundary of this system will be illustrated and the results of Theorem 4.2 will be verified. System (5.1) possesses an asymptoticaly 
stable equilibrium point $x^{s}=(0.35 ; 0.34)$ and two type-1 saddle-node equilibrium points on the stability boundary $\partial A\left(x^{s}\right)$, they are; $q_{1}=(1,42 ; 3,39)$ and $q_{2}=$ $(2,12 ;-3,87)$. Moreover, eight unstable hyperbolic equilibrium points also belong to the stability boundary $\partial A\left(x^{s}\right)$. The stability boundary $\partial A(0,35 ; 0,34)$ is formed, according to Theorem 4.2 , as the union of the manifolds $W^{c^{-}}\left(q_{1}\right), W^{c^{-}}\left(q_{2}\right)$ and the stable manifolds of the unstable hyperbolic equilibrium points that belong to the stability boundary, see Figure 3 .

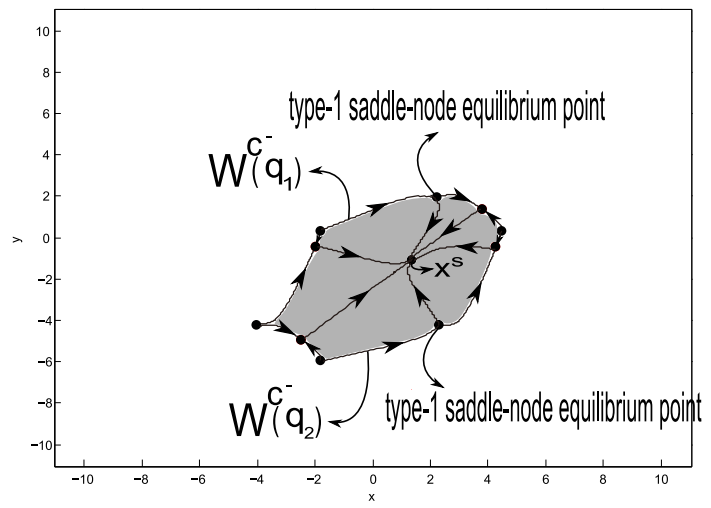

Figure 3: The gray area is the stability region of the asymptoticaly stable equilibrium point $x^{s}$. The stability boundary $\partial A(0,35 ; 0,34)$ is formed by the stable component of the center manifolds of the saddle-node equilibrium points $q_{1}$ and $q_{2}$ union with the stable manifolds of all the unstable hyperbolic equilibrium points that belong to the stability boundary.

\section{Conclusions}

This paper developed the theory of stability region of nonlinear dynamical systems by generalizing the existing results on the characterization of the stability boundary of asymptotically stable equilibrium points. The generalization developed in this paper considers the existence of a particular type of non-hyperbolic equilibrium point on the stability boundary, the so called saddle-node equilibrium point. Necessary and sufficient conditions for a saddle-node equilibrium point lying on the stability boundary were presented. A complete characterization of the stability boundary when the system possesses saddle-node equilibrium points on the stability boundary was developed for a large class of nonlinear dynamical systems. This characterization is an important step to study the behavior of the stability boundary and stability region under parameter variation.

Resumo. Uma caracterização dinâmica da fronteira da região de estabilidade para uma ampla classe de sistemas dinâmicos autônomos é desenvolvida neste artigo. Essa caracterização generaliza os resultados existentes na medida em que permite a existência de pontos de equilíbrio sela-nó na fronteira da região de estabilidade. Mostra-se que a fronteira da região de estabilidade de um ponto de 
equilíbrio assintoticamente estável consiste das variedades estáveis dos pontos de equilíbrio hiperbólicos na fronteira da região de estabilidade e das variedades estáveis, centro-estáveis e centrais dos pontos de equilíbrio sela-nó na fronteira da região de estabilidade.

Palavras-chave. Região de estabilidade, fronteira da região de estabilidade, ponto de equilíbrio sela-nó.

\section{References}

[1] L.F.C. Alberto, H.D. Chiang, Uniform approach for stability analysis of fast subsystem of two-time scale nonlinear systems, Int. J. Bifurcat Chaos Appl. Sci. Eng., 17 (2007), 4195-4203.

[2] F.M. Amaral, L.F.C. Alberto, Stability Boundary Characterization of Nonlinear Autonomous Dynamical Systems in the Presence of a Type-Zero Saddle-Node Equilibrium Point, Tendências em Matemática Aplicada e Computacional, 11 (2010), 111-120.

[3] F.M. Amaral, L.F.C. Alberto, Stability region bifurcations of nonlinear autonomous dynamical systems: type-zero Saddle-node bifurcations, International Journal of Robust and Nonlinear Control, 21, No. 6 (2011), 591-612.

[4] H.D. Chiang, F. Wu, P.P. Varaiya, Foundations of direct methods for power system transient stability analysis, Institute of Electrical and Electronics Engineers Trans. on Circuits and Systems, 34, No. 2 (1987), 160-173.

[5] H.D. Chiang, F. Wu, P.P. Varaiya, Foundations of the potential energy boundary surface method for power transient stability analysis, Institute of Electrical and Electronics Engineers Trans. on Circuits and Systems, 35, No. 6 (1988), 712-728.

[6] H.D. Chiang, M.W. Hirsch, F.F. Wu, Stability region of nonlinear autonomous dynamical systems, Institute of Electrical and Electronics Engineers Trans. on Automatic Control, 33, No. 1 (1988), 16-27.

[7] H.D. Chiang, J.S. Thorp, Stability regions of nonlinear dynamical systems: a constructive methodology, Institute of Electrical and Electronics Engineers Transactions on Automatic Control, 34, No. 12 (1989), 1229-1241.

[8] H.D. Chiang, L. Fekih-Ahmed, Quasi-stability regions of nonlinear dynamical systems: optimal estimations, Institute of Electrical and Electronics Engineers Trans. on Circuits and Systems, 43 , No. 8 (1996), 636-643.

[9] H.D. Chiang, "Direct Methods For Stability Analysis of Electric Power Systems - Theoretical Foundation", Bcu Methodologies, And Applications, John Wiley and Sons, 2010.

[10] R. Genesio, M. Tartaglia, A. Vicino, On the estimation of asymptotic stability regions: State of the art and new proposals, Institute of Electrical and Electronics Engineers Transactions on Automatic Contral, 8 (1985), 747-755. 
[11] J. Guckenheimer, P. Holmes, "Nonlinear Oscilations,Dynamical Systems and Bifurcations of Vector Fields", Springer -Verlag, New York, 1983.

[12] V. Guillemin, A. Pollack, "Differential Topology", Englewood Cliffs, NJ: Prentice-Hall, 1974.

[13] M.W. Hirsch, C.C. Pugh, M. Shub, Invariant manifolds, Bull. Amer. Math. Soc., 76, No. 5 (1970), 1015-1019.

[14] W. Hurewicz., H. Wallman, "Dimension Theory", Princeton, NJ: Princeton University Press, 1948.

[15] P. Kokotovic, R. Marino, On vanishing stability regions in nonlinear systems with high-gain feedback, Institute of Electrical and Electronics Engineers Trans. Automat. Contr., 31, No. 10 (1986), 967-970.

[16] M. Loccufier, E. Noldus, A new trajectory reversing method for estimating stability regions of autonomous dynamic systems, Institute of Electrical and Electronics Engineers Nonlinear Dyn., 21 (2000), 265-288.

[17] R.M. May, "Stability and Complexity in Model Ecosystems", Princeton, NJ: Princeton University Press, 1973.

[18] J. Palis, On morse-smale dynamical systems, Topology, 8, 385-405.

[19] R.M. Peterman, A simple mechanism that causes collapsing stability regions in exploited salmonid populations, J. Fish. Res. Board Can., 34, 1977.

[20] S. Smale, Differentiable dynamical systems, Bull. Amer. Math. Soc., 73 (1967) $747-817$.

[21] J. Sotomayor, Generic bifurcations of dynamical systems. In Dynamical Systems, (1973), 549-560.

[22] S. Wiggins, "Introduction to Applied Nonlinear Dynamical Systems and Chaos", Springer -Verlag, New York, 2003. 